\title{
The Effect of River Flow Retardation on Algal Growth
}

\author{
Hun-Kyun Bae \\ Dept. of Global Environment, School of Environment, Keimyung University, Dalsegu, 704-701, Daegu, S. Korea \\ *Corresponding Author: hunkyunbae@kmu.ac.kr
}

Copyright (C) 2013 Horizon Research Publishing All rights reserved.

\begin{abstract}
How retardation of river flow, resulted from weir construction, affected on algal growth were investigated. 14 sampling points along with The Nakdong River, South Korea, were chosen. Monthly samples were taken from March 2012 to October 2012 to check changes of numbers and types of algae. The dominant algae changed from diatom during spring to blue-green algae during summer. Diatom became a dominant one again for several sampling points by the time which air temperature started to go down, September and then they were replaced as dominant species for most sampling points at October. As results, changes of dominant algae showed seasonal effects and the most important factor for algal growth at this region is the changing pattern of temperature.
\end{abstract}

Keywords Dominant algae, Nakdong River, Seasonal effects, Temperature

\section{Introduction}

Constructing dam or weir is the common way to reserve the water to make up water shortage problems. Those methods, however, might bring ecological problems. Water resources in Korea greatly depend on rivers, but dramatic changes of flow rate between rainy season and dry season makes it difficult to secure water resources [1-4]. Korean government completed nationwide scale construction project, so called 'the four major river project', at the end of 2012 to solve such problems. The main purpose of the project were obtaining water resources and managing water quality throughout building 16 weirs along with four major rivers in Korea. During constructions, even after the project had been completed, strong opinions, which concerns degradation of water quality because those 16 weirs would detain water, kept representing. Especially, one worries rapid algal growth, so called water bloom, because of eutrophication due to detained water at weirs.

The causes of eutrophication would be sewage, waste water, fertilizer from farmland, detention of water flow, etc. and eutrophication would lead water blooms. Water blooms, sometimes, bring serious problem because of toxic blue-green algae such as Microcystis, Nodularia, Anabaena, Oscillatoria, etc. and this severely degrades the quality of water resources [5-9]. Water blooms also cause the disruption of water treatment process by inhibiting the coagulation and filtration processes as well as generating unpleasant taste and odors [10-11]. Any event which human being or wild animals in Korea were damaged by water blooms was not reported, but many were occurred in North America, Australia and Japan [12-15]. However, Arguments for possibilities of such occasions keep raising because river flow retardations due to constructed weirs may bring serious water blooms and water reserved in 16 weirs will be used for drinking water resources for millions of populations.

In this study, therefore, the status of algae after building weirs was investigated focused on middle area of the Nakdong River, longest river and most number of weirs, eight, was built among the four major rivers in Korea.

\section{Materials and Methods}

\subsection{Study area}

Figure 1 shows the study area, locations of weirs and each sampling point. Middle parts of the Nakdong River

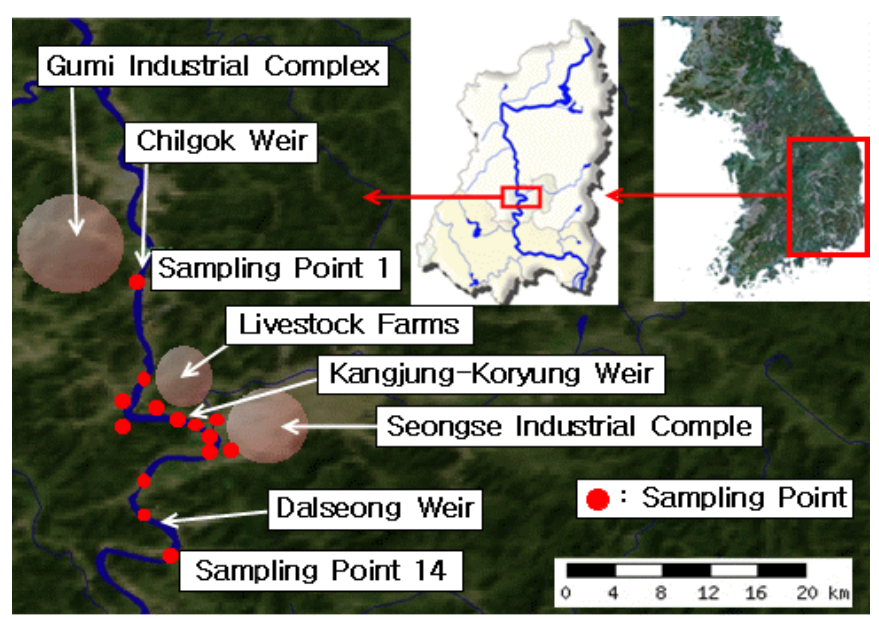

Figure 1. The study area and sampling points 
which were expected to have the most serious water blooms were chosen. 14 sampling points - nine at the main stream and five at branches - were selected for this study. Consecutive umbers were given from sampling site at the upstream to those at downstream, 1 through 14 . Three weirs are located in this study area. Several water pollution sources are located around the study area. For example, Gumi Industrial Complex is located close to sampling point No. 1 and Sungse Industrial Complex is placed at the right side of the Kangjung-Koryung weir. Also, a number of the livestock farms are located at upper side of the Kangjung-Koryung weir, closed to sampling point No. 2 and No. 5.

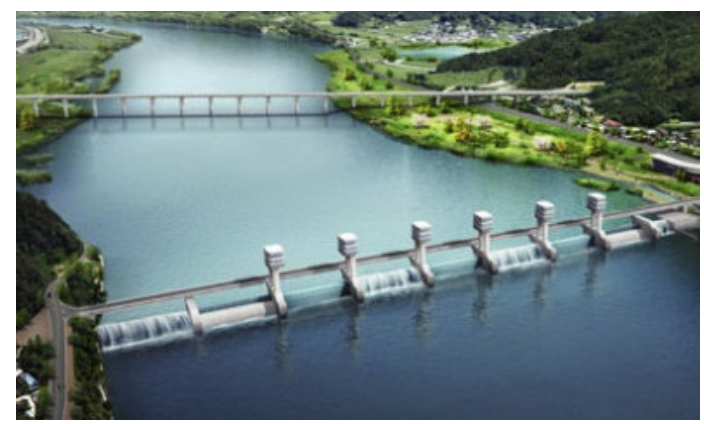

(a) Chilgok Weir

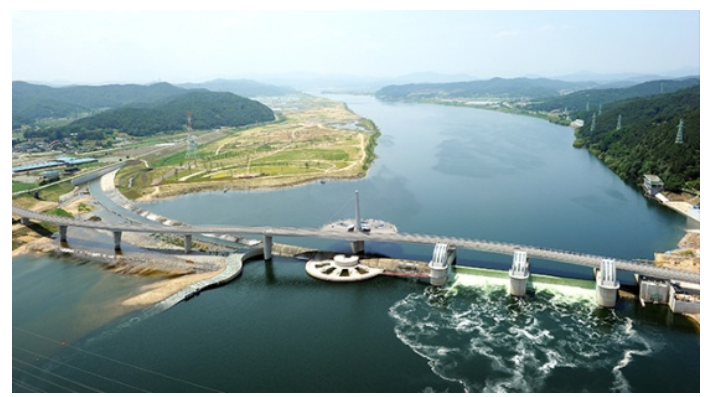

(b) Kangjung-Koryung Weir

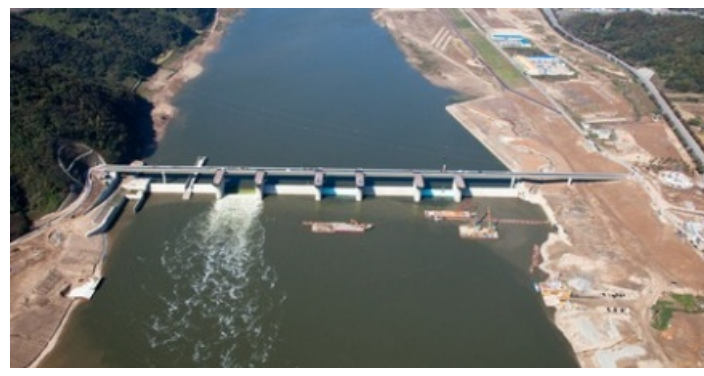

(c) Dalseong Weir

Figure 2. Weirs located in the study area

Table 1. Datailed information for each weir

\begin{tabular}{c||c|c|c}
\hline Weir & Chilgok & Kangjun-Koryung & Dalseong \\
\hline \hline $\begin{array}{c}\text { Total Length } \\
(\mathrm{m})\end{array}$ & 400 & 953 & 580 \\
\hline $\begin{array}{c}\text { Height } \\
(\mathrm{m})\end{array}$ & 12 & 11.5 & 9.5 \\
\hline $\begin{array}{c}\text { Capacity } \\
\left(\text { million } \mathrm{m}^{2}\right)\end{array}$ & 8.9 & 10 & 5.6 \\
\hline
\end{tabular}

Figure 2 shows the view of each weir and Table 1 contains their information. As shown in figure and table, scales of those weirs are big enough to call them a dam which means river flow retardation would be expected.

\subsection{Water samples}

Water samples from 14 sampling points were taken every month from March 2012 to October 2012. Water samples were observed following to Korea Standard Methods for Water Pollution. 2L of water samples at each sampling points were taken and immediately delivered to watershed management lab. at Keimyung University. $500 \mathrm{~mL}$ of water from original water samples were taken after fully agitated and then $1 \sim 2 \mathrm{v} / \mathrm{v} \%$ of rugol solution were added. After 24 hours, supernatants were removed to get concentrated water samples. A hemocytometer was used to count the number of algae with 200 to 1000 times of microscope. Counting repeated dozens of times for each sample.

\section{Results}

Figure 3 shows examples of water bloom at sampling point 3 and sampling point 6 . Pictures were taken on July $3^{\text {th }}$ which day had a high temperature. Water blooms were occurred in two sampling points out of 14 sampling sites on July $3^{\text {th }}$. Possibilities of water bloom in other sampling points are high because of high temperature during July, but it could not be checked since the study performed a monthly sampling.
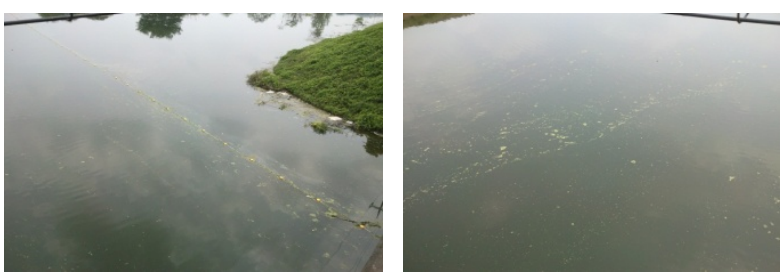

(a) Sampling point 3
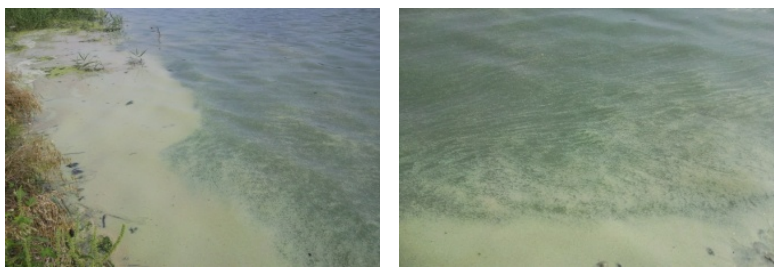

(b) Sampling point 6

Figure 3. Examples of water bloom at some sampling points

Table2 shows changes of dominant algae for each month. Dominant algae from March to May which months had relatively low temperature levels were diatoms, cyclotella spp., especially during March, dominant algae for all sampling points were cyclotella spp. Temperature at the study area became to rise from June 2012 and dominant algae changed for several sampling point, diatom to 
blue-green algae and from July 2012, most of sampling points were dominated by blue-green algae such as Microcystis spp., Anabaena sp. and Oscillatoria spp. which species are toxic to human being or wild animals. However, diatom would be the main species for several sampling points from September since air temperature became to go down and dominant algae at most of sampling points were diatom. Also, water blooms did not occur at most of sampling sites throughout whole year, except July and August. Rapid algal growths were shown during May and June when air temperature started to rise, but water blooms were not happened because of rainfall events.

Table 2. Monthly changes of dominant algae at each site

\begin{tabular}{|c|c|c|c|c|c|c|c|c|}
\hline $\begin{array}{c}\text { Sampling } \\
\text { point }\end{array}$ & Mar. & Apr. & May & Jun. & Jul. & Aug. & Sep. & Oct. \\
\hline 1 & $\begin{array}{l}\text { Cyclotella } \\
\text { spp. }\end{array}$ & Cyclotella spp. & $\begin{array}{c}\text { Synedra } \\
\text { acus }\end{array}$ & $\begin{array}{c}\text { Pediastrum } \\
\text { sp. }\end{array}$ & $\begin{array}{l}\text { Microcystis } \\
\text { spp. }\end{array}$ & $\begin{array}{l}\text { Oscillatoria } \\
\text { spp. }\end{array}$ & $\begin{array}{l}\text { Nitzschia } \\
\text { fonticola }\end{array}$ & $\begin{array}{c}\text { Aulacoseira } \\
\text { granulata }\end{array}$ \\
\hline 2 & $\begin{array}{c}\text { Cyclotella } \\
\text { spp. }\end{array}$ & Naviculaspp. & $\begin{array}{c}\text { Synedra } \\
\text { acus }\end{array}$ & $\begin{array}{l}\text { Pediastrum } \\
\text { sp. }\end{array}$ & $\begin{array}{c}\text { Scenedesmus } \\
\text { sp. }\end{array}$ & $\begin{array}{l}\text { Scenedesmus } \\
\text { sp. }\end{array}$ & $\begin{array}{l}\text { Fragilaria } \\
\text { crotonensis }\end{array}$ & $\begin{array}{c}\text { Aulacoseira } \\
\text { granulata }\end{array}$ \\
\hline 3 & $\begin{array}{c}\text { Cyclotella } \\
\text { spp. }\end{array}$ & Cyclotella spp. & - & $\begin{array}{c}\text { Aulacoseira } \\
\text { granulata }\end{array}$ & $\begin{array}{c}\text { Anabaena } \\
\text { sp. }\end{array}$ & $\begin{array}{c}\text { Anabaena } \\
\text { sp. } \\
\end{array}$ & $\begin{array}{c}\text { Achnanthes } \\
\text { spp. }\end{array}$ & $\begin{array}{c}\text { Aulacoseira } \\
\text { granulata }\end{array}$ \\
\hline 4 & $\begin{array}{c}\text { Cyclotella } \\
\text { spp. }\end{array}$ & Scenedesmussp. & $\begin{array}{c}\text { Synedra } \\
\text { acus }\end{array}$ & $\begin{array}{c}\text { Aulacoseira } \\
\text { granulata }\end{array}$ & $\begin{array}{c}\text { Lepocinclis } \\
\text { spp. }\end{array}$ & $\begin{array}{c}\text { Aulacoseira } \\
\text { granulata }\end{array}$ & $\begin{array}{l}\text { Nitzschia } \\
\text { fonticola }\end{array}$ & $\begin{array}{c}\text { Cryptominas } \\
\text { spp. }\end{array}$ \\
\hline 5 & $\begin{array}{l}\text { Cyclotella } \\
\text { spp. }\end{array}$ & Cyclotella spp. & $\begin{array}{c}\text { Pediastrum } \\
\text { sp. }\end{array}$ & $\begin{array}{c}\text { Pediastrum } \\
\text { sp. }\end{array}$ & $\begin{array}{c}\text { Oscillatoria } \\
\text { spp. }\end{array}$ & $\begin{array}{l}\text { Oscillatoria } \\
\text { spp. }\end{array}$ & Nitzschia palea & $\begin{array}{c}\text { Aulacoseira } \\
\text { granulata }\end{array}$ \\
\hline 6 & $\begin{array}{c}\text { Cyclotella } \\
\text { spp. }\end{array}$ & Cyclotella spp. & $\begin{array}{l}\text { Fragilaria } \\
\text { crotonensis }\end{array}$ & $\begin{array}{c}\text { Aulacoseira } \\
\text { granulata }\end{array}$ & $\begin{array}{c}\text { Microcystis } \\
\text { spp. }\end{array}$ & $\begin{array}{l}\text { Volvox } \\
\text { aureus }\end{array}$ & Scenedesmussp. & $\begin{array}{c}\text { Aulacoseira } \\
\text { granulata }\end{array}$ \\
\hline 7 & $\begin{array}{c}\text { Cyclotella } \\
\text { spp. }\end{array}$ & Cyclotella spp. & $\begin{array}{c}\text { Cyclotella } \\
\text { spp. }\end{array}$ & $\begin{array}{c}\text { Cyclotella } \\
\text { spp. }\end{array}$ & $\begin{array}{c}\text { Cyclotella } \\
\text { spp. }\end{array}$ & $\begin{array}{c}\text { Cyclotella } \\
\text { spp. }\end{array}$ & $\begin{array}{c}\text { Achnanthes } \\
\text { spp. }\end{array}$ & $\begin{array}{c}\text { Aulacoseira } \\
\text { granulata }\end{array}$ \\
\hline 8 & $\begin{array}{c}\text { Cyclotella } \\
\text { spp. }\end{array}$ & Cyclotella spp. & - & Pediastrumsp. & $\begin{array}{c}\text { Anabaena } \\
\text { sp. }\end{array}$ & $\begin{array}{c}\text { Aulacoseira } \\
\text { granulata }\end{array}$ & - & $\begin{array}{c}\text { Cyclotella } \\
\text { spp. }\end{array}$ \\
\hline 9 & $\begin{array}{c}\text { Cyclotella } \\
\text { spp. }\end{array}$ & Cyclotella spp. & $\begin{array}{l}\text { Fragilaria } \\
\text { crotonensis }\end{array}$ & Pediastrumsp. & $\begin{array}{l}\text { Oscillatoria } \\
\text { spp. }\end{array}$ & $\begin{array}{c}\text { Microcystis } \\
\text { spp. }\end{array}$ & $\begin{array}{l}\text { Nitzschia } \\
\text { fonticola } \\
\end{array}$ & $\begin{array}{c}\text { Cyclotella } \\
\text { spp. }\end{array}$ \\
\hline 10 & - & - & - & - & $\begin{array}{c}\text { Coelastrum } \\
\text { sp. }\end{array}$ & $\begin{array}{c}\text { Oscillatoria } \\
\text { spp. }\end{array}$ & $\begin{array}{c}\text { Achnanthes } \\
\text { spp. }\end{array}$ & $\begin{array}{l}\text { Oscillatoria } \\
\text { spp. }\end{array}$ \\
\hline 11 & $\begin{array}{l}\text { Cyclotella } \\
\text { spp. }\end{array}$ & Cyclotella spp. & $\begin{array}{c}\text { Synedra } \\
\text { acus }\end{array}$ & $\begin{array}{c}\text { Cyclotella } \\
\text { spp. }\end{array}$ & $\begin{array}{c}\text { Cyclotella } \\
\text { spp. }\end{array}$ & $\begin{array}{c}\text { Microcystis } \\
\text { spp. }\end{array}$ & 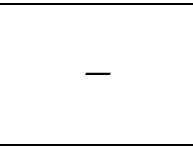 & $\begin{array}{c}\text { Aulacoseira } \\
\text { spp. } \\
\text { Cyclotella } \\
\text { spp. }\end{array}$ \\
\hline 12 & $\begin{array}{c}\text { Cyclotella } \\
\text { spp. }\end{array}$ & Cyclotella spp. & $\begin{array}{c}\text { Pediastrum } \\
\text { sp. }\end{array}$ & $\begin{array}{c}\text { Aulacoseira } \\
\text { granulata }\end{array}$ & $\begin{array}{c}\text { Oscillatoria } \\
\text { spp. }\end{array}$ & $\begin{array}{l}\text { Oscillatoria } \\
\text { spp. }\end{array}$ & $\begin{array}{c}\text { Asterionella } \\
\text { formosa }\end{array}$ & $\begin{array}{c}\text { Aulacoseira } \\
\text { granulata }\end{array}$ \\
\hline 13 & $\begin{array}{c}\text { Cyclotella } \\
\text { spp. }\end{array}$ & Cyclotella spp. & $\begin{array}{c}\text { Coelastrum } \\
\text { sp. }\end{array}$ & $\begin{array}{c}\text { Aulacoseira } \\
\text { granulata }\end{array}$ & $\begin{array}{c}\text { Oscillatoria } \\
\text { spp. }\end{array}$ & $\begin{array}{c}\text { Microcystis } \\
\text { spp. }\end{array}$ & $\begin{array}{l}\text { Nitzschia } \\
\text { fonticola }\end{array}$ & $\begin{array}{c}\text { Aulacoseira } \\
\text { granulata }\end{array}$ \\
\hline 14 & $\begin{array}{c}\text { Cyclotella } \\
\text { spp. }\end{array}$ & Cyclotella spp. & $\begin{array}{l}\text { Fragilaria } \\
\text { crotonensis }\end{array}$ & $\begin{array}{c}\text { Pediastrum } \\
\text { sp. }\end{array}$ & $\begin{array}{c}\text { Oscillatoria } \\
\text { spp. }\end{array}$ & $\begin{array}{l}\text { Oscillatoria } \\
\text { spp. }\end{array}$ & $\begin{array}{l}\text { Nitzschia } \\
\text { fonticola }\end{array}$ & $\begin{array}{c}\text { Cyclotella } \\
\text { spp. }\end{array}$ \\
\hline
\end{tabular}

As results, the changes of dominant species depend on temperature rather than river flow retardation. Temperature also plays an important role for water blooms. Previous studies also provided the importance of temperature [16-18]. However, rainfall events could be the main factor whether water blooms occur for this study.

Figure 4 shows pictures of dominant algae of each sampling points for this study. 


\section{Conclusion}

In this study partial sections of the Nakdong River were chosen to investigate the status of algae after major weir constructions. Results from monthly monitoring for dominant algae indicated that algal growth greatly depends on temperature. Diatom were the main species from March to May which temperatures were relatively low. Blue-green algae were dominant for all sites during July and August and water blooms were happened during this high temperature seasons, but dominant algae were replaced from blue-green algae to diatom as air temperature went down and no more water bloom occurred. As a result, algal growth was dependent on temperature rather than detention of water flow.
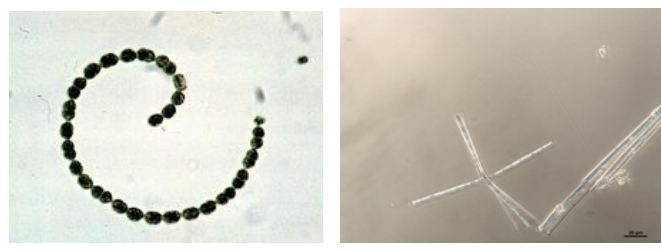

Anabena

Asterionella Formosa

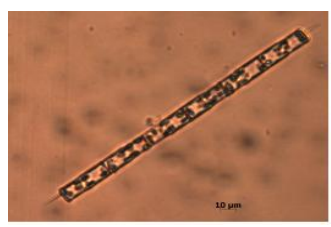

Aulacoseira granulata

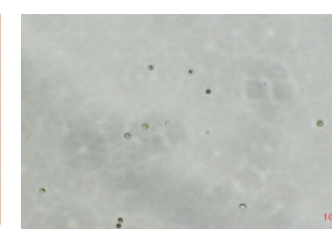

Choloella spp.
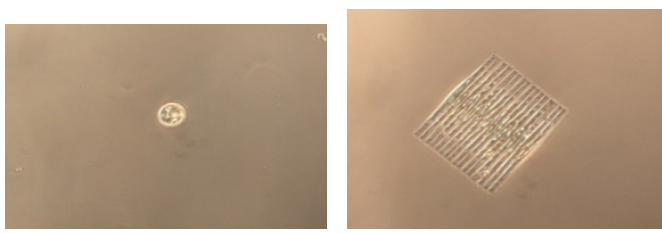

Cyclotella sp.

Fragilaria construens

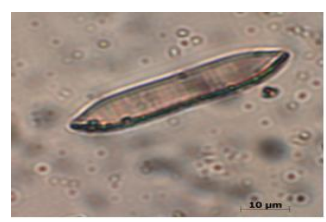

Nitzschia palea

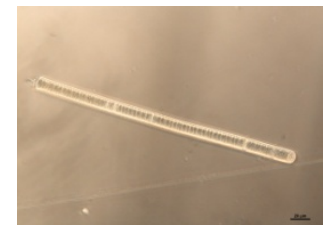

Oscillatoria spp.

Figure 4. Pictures of dominant algae

\section{Acknowledgements}

The study was funded by 2012 Daegu Green Environment Center Research Funds.

\section{REFERENCES}

[1] Y. T. Kang. Developing advanced waste water treatment systems for recovering Nakdong River, J. of Korean Soc. of Wat. Sci. and Tech., Vol.3, No.4, 21-30, 1995.

[2] Y. T. Kang, K. S. Hyun, H. C. Yang. Predicting and managing water quality fir Nakdong River, J. of Korean Soc. of Wat. Sci. and Tech., Vol.4, No.3, 3-15, 1996.

[3] J. M. Lee, C. G. Park, C. Kim. Distributions and contaminations of Boron at Nakdong River, , J. of Korean Soc. of Wat. Enviro., Vol.21, No.3, 236-241, 2005.

[4] I. D. Jung, Current and future status of water quality for Nakdong River, Mater Thesis Hanyang University, Korea, 1996.

[5] B. Y. Park, J. Choi, S. M. Jang. Environment Science, Hyungseol press, Korea, 1996.

[6] B. S. Son. Introduction of Environment Science, Shingang press, 1995.

[7] G. C. Gerloff, G. P. F. Fitzgerald, F. Skoong. The mineral of Microcystis aeruginosa, Am. J. Bot. 39(1), 26-32, 1995.

[8] A. Zehnderm, P. R. Gorham. Factors influencing the growth of Microcystis aeruginosa, Can. J. Microbiol. 6(6), 645-662, 1960 .

[9] B.-L. Yuan, J.-H. Qu, M.-L. Fu. Removal of cyanobacterial microcystin-LR by ferrate oxidation-coagulation, Toxicon 40(8), 1129-1134, 2002.

[10] R. I. Daly, L. Ho, J. D. Brookers. Effect of chlorination on Microcystis aeruginosa cell integrity and subsequent microcystin release and degradation, Environ. Sci. Technol. 41(12), 4447-4453, 2007

[11] T. Takaara, D. Sano, H. Konno, T. Omura. Cellular proteins of Microcystis aeruginosa inhibiting coagulation with polyaluminum chloride, Wat. Res. 41(8), 1653-1658, 2007.

[12] I. R. Falconer, A. M. Bersford, M. T. C. Runnegar. Evidence of liver damage by toxin from a bloom of the blue-green alga, Microcystis aeruginosa, Med. J. Aust. 1(11), 511-514, 1983.

[13] W. W. Carmichael, R. S. Saffermann. A status report on planktonic cyanobacteria(blue-green algae) and their toxins. EPA/600/R 92/079, 1992.

[14] K. Sivonen, M. Namikoshi, W. R. Carmichael, F. Sun, L. Rouhiainen, R. Luukkainen, K. L. Rinehart. Isolation and characterization of a variety of microcystins from seven strains of the cyanobacterial genus Anabaena, Appl. Environ. Microbiol. 58(8), 2495-2500, 1992.

[15] R. Luukkainen, K. Sivonen, M. Namikoshi, M. Fardig, K. L. Rinehart, S. I. Niemela. Isolation and identification of eight microcystin from thirteen Oscillatoria agardhii strains and structure of a new microcystin. Appl. Environ. Microbiol. 59(7), 2204-2209, 1993.

[16] Z. Chu, X. Jin, N. Iwami, Y. Inamori. The effect of temperature on growth characteristics and competitions of Microcystis aeruginosa and Oscillatoria mougeotii in a shallow, eutrophic lake simulator system, Hydrobiology 194, 217-223, 2007.

[17] E. Fouilland, R. J. G. Leakey, K. Jones, J. Slater, I. A. 
Calleja, The response of a planktonic microbial community to experimental simulations of sudden mixing conditions in temperate coastal waters: Importance of light regime and nutrient enrichment, J. of Experi. Mar. Bio. And Eco. 351(1-2), 211-225, 2007.
[18] T. W. Davis, D. L. Berry, G. L. Boyer, C. J. Gobler. The effects of temperature and nutrients on the growth and dynamics of toxic and non-toxic strains of Microcystis during cyanobacteria blooms, Harmful Algae 8(5), 715-725, 2009. 